\title{
Influência do tratamento térmico e da acidez no comportamento reológico de amidos nativos funcionais de milho cerosos orgânicos comerciais
}

\author{
Heat treatment and acidity influence on the rheological behavior of commercial organic waxy corn starch
}

Lizielle Maria Ricardo GUERREIRO ${ }^{1 \star}$, Florencia Cecilia MENEGUELLI ${ }^{1}$

\begin{abstract}
Resumo
Os amidos são amplamente utilizados em alimentos como molhos para salada, molhos e pratos prontos. Entretanto, as propriedades funcionais de amidos nativos não resistem aos processos estressantes tais como tratamento térmico, acidez e alto cisalhamento. Os amidos podem ser modificados quimicamente neste sentido, mas não atribuem ao alimento o rótulo de "natural". Uma outra opção é obter amidos naturais resistentes às condições de estresse. O objetivo do presente trabalho foi a avaliação de dois amidos que se comercializam rotulados como nativos e orgânicos frente ao tratamento térmico e à acidez. Suspensões dos amidos nativos funcionais orgânicos (9460 e 9560, National Starch and Chemical Industrial) preparadas numa concentração de $5 \%$ (peso/volume), foram acidificadas com ácido cítrico $1 \mathrm{M}$ ou autoclavadas a $121^{\circ} \mathrm{C}$ por 30 minutos. O resultado dos tratamentos foi avaliado por microscopia óptica, pelas curvas de escoamento e pelos espectros mecânicos, obtidos por reologia estacionária e dinâmica. A acidez e o tratamento térmico aumentaram a estruturação dos géis dos amidos, que resistiram aos processos estressantes. Os géis apresentaram comportamento não newtoniano, (pseudoplástico) e tixotrópico. O comportamento pode ser modelado pela equação Lei da Potência ou Herschel-Bukley. Todos os géis apresentaram comportamento viscoelástico de gel fraco que foi preservado nos diversos tratamentos.
\end{abstract}

Palavras-chave: amido; milho; ceroso; funcional; tratamento térmico; acidez.

\begin{abstract}
Starches are widely utilized in foodstuffs as salad dressing, sauce, and processed foods. However, the functional properties of native starches are affected by processes such as heat treatment, acidity, and high shear stress. Starches can be modified chemically in order to have characteristics required by industries, but not to attribute the food a "natural and safe" label. Another option is to obtain native starches resistant to food processing stress conditions. The objective of this paper is the evaluation of commercial native organic starches under food processing stress conditions such as heat treatment and acidity. Suspensions of native functional organic starches ( 9460 and 9560 , National Starch, and Chemical Industry) were prepared at $5 \%$ concentration (w/v), acidified with $1 \mathrm{M}$ citric acid, or autoclaved at $121{ }^{\circ} \mathrm{C}$ for $30 \mathrm{minutes}$. The results were evaluated by optical microscopy, flow curve, and viscoelastic aspects which were obtained by steady and oscillatory rheology. The acidity and heat treatment increased the starch gels structure that resisted to the processing stress conditions. The starch gels exhibited non-newtonian (pseudoplastic) and thixotropic behavior. Flow data were fitted to the Power Law or Herschel-Bukley models. All samples exhibited gel-like viscoelastic behavior which was preserved under several stress conditions.
\end{abstract}

Keywords: starch; corn; waxy; functional; heat treatment; acidity.

\section{Introdução}

Produtos elaborados, como molhos para salada, conservas, sobremesas, pratos prontos, dentre outros, passam por alguma etapa de bombeamento, esterilização, congelamento ou acidificação, durante o processamento. Um aditivo amplamente empregado nestes alimentos é o amido, utilizado na indústria de alimentos como agente espessante e gelificante (KUHN; SCHLAUCH, 1994; MARQUES et al., 2006). Para resistir a estas condições estressantes, os amidos devem conservar suas propriedades funcionais como a textura inicial, não liberar água nem alterar cor, preservar aromas, resistir a $\mathrm{pH}$ baixo e a esterilização e ainda aumentar a vida de prateleira do produto. Em geral, o uso de amido nativo é limitado devido às perdas ou ao decréscimo de propriedades funcionais apresentando uma baixa resistência à tensão de cisalhamento, uma propensão à decomposição térmica e uma alta retrogradação e sinérese, que podem ocorrer durante ou após o processamento. Essas falhas podem ser superadas pela modificação do amido por métodos químicos, físicos ou enzimáticos. Os amidos são constituídos essencialmente por $2 \alpha$-poliglucanas, a amilose e a amilopectina. A amilose, que é uma molécula essencialmente linear de ligações $(1 \rightarrow 4)-\alpha$-D-glucose com um pequeno grau de ramificações (TAKEDA et al., 1987). A amilopectina é uma molécula altamente ramificada, na qual cadeias de $(1 \rightarrow 4)-\alpha-D-$ glucanas são conectadas por ligações $\alpha(1 \rightarrow 6)$ (ELLIS et al., 1998) com cadeias curtas como dupla hélice em clusters de caráter parcialmente cristalino (ZOBEL, 1988, SINGH et al., 2003). $\mathrm{O}$ amido, quando aquecido em excesso de água, gelatiniza, passando por uma fase de transição de ordem-desordem, em que ocorrem a hidratação, a expansão e o rompimento granular e, ainda, a solubilização das moléculas de amido. Os géis de

Recebido para publicação em 29/10/2007

Aceito para publicação em 4/1/2009 (002969)

Departamento de Engenharia de Alimentos, Faculdade de Engenharia de Alimentos, Universidade Estadual de Campinas - UNICAMP, CP 6121, CEP 13083-862,

Campinas - SP, Brasil, E-mail: fcm@fea.unicamp.br

${ }^{*}$ A quem a correspondência deve ser enviada 
amido formados durante a gelatinização não são estáveis. Por esfriamento e/ou por armazenamento produzem-se diferentes transformações estruturais que, em seu conjunto, se definem como retrogradação (SANDERSON, 1981). Para se controlar propriedades reológicas finais de produtos alimentícios à base de amido, é necessário ter um bom conhecimento da influência da temperatura e seus efeitos no comportamento reológico do amido nos vários passos do processo (LAGARRIGUE; ALVAREZ, 2001).

Substâncias ácidas são adicionadas em muitos alimentos como acidulantes ou conservadores. Os ácidos primeiramente hidrolisam a região amorfa antes de atacarem as regiões cristalinas, sendo a amilose e amilopectina hidrolisadas simultaneamente a moléculas menores (WANG; WANG, 2001). Hirashima, Takahashi, Nishinari (2005) estudaram o comportamento reológico de amido de milho em diferentes $\mathrm{pH}$ e diferentes ácidos adicionados antes e depois da gelatinização. Eles concluíram que a viscosidade é uma função do pH e não depende do tipo de ácido. Os valores de viscosidade aparente, numa taxa de deformação de $10 \mathrm{~s}^{-1}$, entre $\mathrm{pH}$ 5,6 e 3,6 foram maiores que o controle. A mudança da viscosidade foi induzida por uma mudança no tamanho do grânulo de amido e pelos números de cadeias de amilose e amilopectina causada pela adição de ácido. Um aumento na viscosidade nunca foi relatado na literatura anteriormente. Muitas pesquisas relataram que a viscosidade da pasta de amido decresce na presença de ácidos (VALLÉS-PÀMIES et al., 1997; SRIBURI; HILL; MITCHELL, 1999; SRIBURI; HILL, 2000). Yamada, Morimoto, Hisamatsu (1986) relataram que a viscosidade da pasta de fécula de batata diminuiu ajustando-se o $\mathrm{pH}$ com ácido cítrico ou acético, enquanto que a pasta de amido de milho não foi afetada pelos ácidos.

Os amidos da linha "Novation" 9460 e 9560 são amidos nativos funcionais que possuem como base um amido de milho ceroso com propriedades similares às dos amidos modificados, segundo informa o fabricante. Ambos os amidos são recomendados para aplicações em condimentos, sopas e molhos de salada e produtos lácteos, pois apresentam uma tensão crítica ou residual apreciável e podem ser utilizados sem necessidade de aquecimento. $\mathrm{O}$ amido 9460, além dos usos indicados acima, também é indicado para uso de panificação, como para misturas prontas de bolos, muffins, snacks, brownies.

Assim, inserido neste contexto, este trabalho tem como objetivo estudar o comportamento reológico das suspensões destes amidos comerciais, considerados amidos funcionais, em condições de acidez e de tratamento térmico.

\section{Material e métodos}

\subsection{Matéria-prima}

As matérias-primas utilizadas neste trabalho foram os amidos pré-gelatinizados de milho cerosos orgânicos "Novation" 9560 e 9460 fornecidos pela filial brasileira da National Starch and Chemical Industrial Ltda.

\subsection{Microscopia óptica dos amidos}

Suspensões contendo $1 \mathrm{~g}$ dos amidos comerciais 9460 e 9560 em $100 \mathrm{~mL}$ de água destilada foram aquecidas na temperatura de $95^{\circ} \mathrm{C}$ num banho-maria por 1 hora. As amostras com $\mathrm{pH}$ 3,5 foram acidificadas antes de serem aquecidas, num agitador com pHgâmetro (marca Analyser modelo pH 300) utilizando-se ácido cítrico $1 \mathrm{M}$, preparado com ácido cristalizado da marca Synth. Depois de resfriados em temperatura ambiente, uma parte dos géis naturais foi autoclavada a $121^{\circ} \mathrm{C}$ por 30 minutos e novamente resfriada. Os géis foram corados com lugol (solução contendo $2 \mathrm{~g}$ de iodeto de potássio com $0,2 \mathrm{~g}$ de iodo dissolvidos em $100 \mathrm{~mL}$ de água destilada) e uma alíquota foi colocada numa lâmina de vidro e coberta por uma lamínula. A amilose se colore de azul e a amilopectina de um tom avermelhado.

As amostras foram observadas num Microscópio Óptico (HIRASHIMA; TAKAHASHI; NISHINARI, 2005) Carl Ziess modelo Variant Jenamed MF-AKS $24 \times 36$ EXPOMET (Zeiss - Alemanha), com lente de aumento de 40×. Acoplada ao microscópio, uma câmera fotográfica digital (Kodak modelo Easyshare DX4530, resolução de $2580 \times 1932$ (5,0M) pixels), com zoom de $10 \times$, capturava as imagens observadas no microscópio, num aumento total de $400 \times$.

\subsection{Caracterização química do amido}

As análises químicas foram teor de umidade (AOAC, 1997), de cinzas (AOAC, 1997), de lipídios totais, método Soxlet (AOAC 1997), de proteínas, método Kjedahl (AOAC, 1997), de fibras (AOAC, 1975) e de amilose (JULIANO, 1971, modificado por MARTINEZ; CUEVAS, 1989). Todas as análises foram realizadas em triplicata.

\subsection{Preparo dos géis}

Suspensões dos amidos foram preparadas a 5\% de concentração em água destilada. Uma parte destas suspensões foi acidificada com ácido cítrico, da marca Synth, $1 \mathrm{M}$, baixando o $\mathrm{pH}$ para 3,5. Em seguida, as suspensões foram aquecidas em banho-maria a $90{ }^{\circ} \mathrm{C}$ por 30 minutos e resfriadas em temperatura ambiente. Uma parte das amostras com $\mathrm{pH}$ natural foram autoclavadas a $121{ }^{\circ} \mathrm{C}$ por 30 minutos (BRETON-DOLLET, 1996; NURUL; AZEMI; MANAN, 1999) e resfriadas, novamente, em temperatura ambiente.

\subsection{Medidas reológicas}

Os testes foram realizados em um reômetro de tensão controlada, marca Carri Med, modelo $500 \mathrm{CSL}^{2}$. A temperatura de medida foi mantida na placa inferior pelo sistema Peltier. A geometria utilizada foi cone-placa, com $60 \mathrm{~mm}$ de diâmetro e ângulo de $2^{\circ}$. Todas as análises foram feitas em triplicata.

\section{Curvas de escoamento (ensaios estacionários)}

As curvas de escoamento foram determinadas por três rampas contínuas consecutivas de varredura de taxa de deformação de 0 a $300 \mathrm{~s}^{-1}$ com ciclo ascendente, descendente e ascendente, para avaliar e eliminar a tixotropia.

A modelagem matemática foi realizada com os dados da terceira rampa ascendente, após a eliminação da tixotropia das amostras, utilizando o modelo de Herschel-Bulkley $\left(\sigma=\sigma_{\mathrm{o}}+\mathrm{k}(\dot{\gamma})^{\mathrm{n}}\right)$. 
Este modelo é considerado um modelo geral, pois se aplica à maioria dos fluidos, sendo o modelo Lei da Potência (ou Ostwald-de-Waele) um caso particular da equação de HB, com tensão crítica nula. Os valores dos parâmetros foram estimados utilizando o "Solver" do software Excel 2000. Foi possível estimar os valores dos parâmetros, $\mathrm{n}$ (índice de comportamento de escoamento), $\mathrm{k}$ (índice de consistência) e $\sigma_{\mathrm{o}}$ (tensão residual) das equações, além do coeficiente de correlação $\mathrm{R}^{2} \mathrm{e}$ a somatória dos erros $\left(\chi^{2}\right)$.

\section{Espectros mecânicos}

Os espectros mecânicos das diversas amostras foram determinados por varredura da frequência entre 0,01 e $10 \mathrm{~Hz}$, à tensão constante e temperatura de $30^{\circ} \mathrm{C}$, dentro do intervalo linear. $\mathrm{O}$ intervalo linear de resposta, para cada amostra, foi medido realizando uma varredura de tensão, a uma frequência constante de $1 \mathrm{~Hz}$. As tensões utilizadas nas varreduras de frequência para o amido 9460 foram $0,1 \mathrm{~Pa}$, para $\mathrm{pH}$ natural, $0,6 \mathrm{~Pa}$, para $\mathrm{pH}$ ácido e $0,8 \mathrm{~Pa}$ para o tratamento térmico. Para o amido 9560 foram utilizadas 0,6 Pa para $\mathrm{pH}$ natural e ácido e 0,2 Pa para o tratamento térmico.

Os dados reológicos foram analisados estatisticamente utilizando o Teste de Tuckey através do software Mini Tab 14.0.

\section{Resultados e discussão}

\subsection{Composição química}

A composição dos amidos comerciais é apresentada na Tabela 1.

Podemos observar um baixo conteúdo de amilose que o qualificaria como ceroso, entretanto ambos os amidos possuem um conteúdo superior ao informado por Singh et al. (2003), que foi de 1,4 a $2,7 \%$.

\subsection{Microscopia óptica dos amidos}

A microscopia óptica pode ser utilizada como uma ferramenta para elucidar as mudanças estruturais decorrentes do aquecimento ou do uso de um meio ácido, nos grânulos de amido (Figuras 1 e 2). Podemos observar que os grânulos do amido apresentaram-se com formato disforme já na temperatura de $55^{\circ} \mathrm{C}$, pois os amidos são comercializados pré-gelatinizados. Os grânulos dos amidos 9560 e 9460 apresentaram-se muito parecidos tanto no formato, como na coloração, apresentando poucas partes coloridas de azul, pequenos traços de amilose, conforme foi constatado na análise de composição química. Podemos observar que a amilose foi lixiviada dos grânulos, visto que a coloração azul se localiza fora do granulo e estes estão coloridos de vermelho (amilopectina).

Tabela 1. Composição físico-química dos amidos comerciais em (g/100g base seca).

\begin{tabular}{cccccc}
\hline & $\begin{array}{c}\text { Umidade } \\
(\%)\end{array}$ & $\begin{array}{c}\text { Proteína } \\
(\%)\end{array}$ & $\begin{array}{c}\text { Cinzas } \\
(\%)\end{array}$ & $\begin{array}{c}\text { Lipídios } \\
(\%)\end{array}$ & $\begin{array}{c}\text { Amilose } \\
(\%)\end{array}$ \\
\hline 9460 & $6,74 \pm 0,08$ & $0,17 \pm 0,03$ & $0,02 \pm 0,02$ & $0,35 \pm 0,29$ & $9,05 \pm 0,20$ \\
9560 & $7,74 \pm 0,18$ & $0,12 \pm 0,08$ & $0,02 \pm 0,01$ & $0,33 \pm 0,02$ & $7,11 \pm 0,21$ \\
\hline
\end{tabular}

Comparando-se as Figuras 1 e 2 (A, B, C e D), pode-se concluir que não houve mudança significativa da estrutura dos grânulos de amido, que resistiram à ação do ácido cítrico e ao tratamento térmico. A estrutura foi mantida, mas podemos observar uma pequena expansão dos grânulos após o aquecimento e o tratamento térmico e a acidificação do meio. Estes resultados coincidem com os obtidos por Nayouf et al. (2003) que constataram um aumento do diâmetro médio dos grânulos em função do aumento da temperatura de tratamento de 90 a $130{ }^{\circ} \mathrm{C}$.

\subsection{Reologia do amido}

O objetivo destas análises foi avaliar os amidos comerciais reologicamente, visando o conhecimento desses amidos cerosos, com propriedades similares aos amidos modificados, aplicados em diversos produtos alimentícios industrializados.

\section{Ensaios estacionários}

Nas Figuras 3 e 4 apresentam-se as curvas de escoamento dos géis dos amidos comerciais 9460 e 9560, respectivamente. São apresentados os dados das três varreduras de taxas de deformação em forma ascendente e descendente. A diferença entre as curvas da subida 1 e 2 dos géis dos amidos, nos mostram uma quebra de estrutura com a aplicação do cisalhamento, indicando a presença
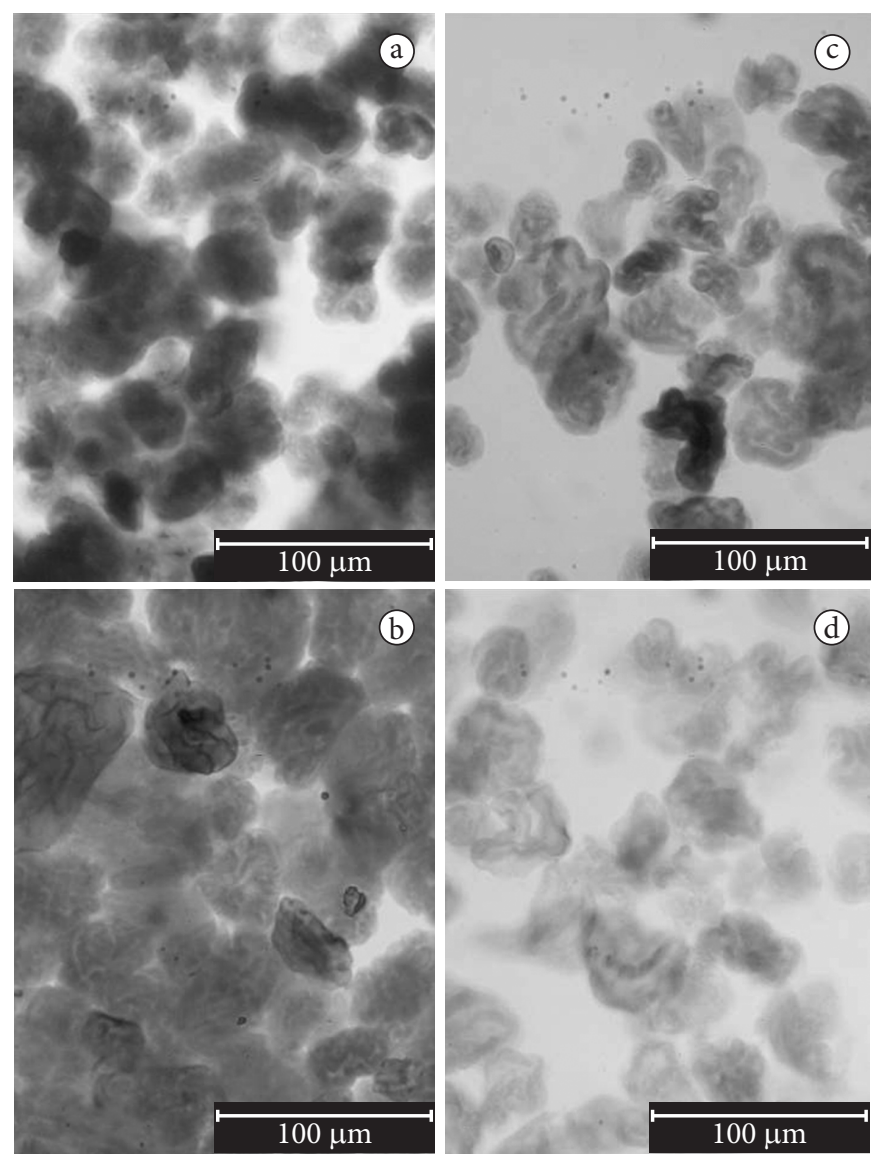

Figura 1. Grânulos dos amidos 9460 na temperatura de $55^{\circ} \mathrm{C}$ (a), aquecidos na temperatura de $95^{\circ} \mathrm{C}(\mathrm{b})$, na temperatura de $95^{\circ} \mathrm{C}$ em pH ácido (c) e depois de autoclavado (d). 
de tixotropia, característica de grande parte dos fluídos. Os dados de tixotropia estão apresentados na Tabela 2.

Suspensões de amido podem apresentar características tixotrópicas quando em concentrações baixas, mas serem reopéticas na medida em que se aumenta a concentração da suspensão ou ainda apresentar as duas características no mesmo ensaio (TATTIYAKUL; RAO, 2000).

Comparando-se as Figuras 3 e 4, podemos observar que a suspensão de amido 9560 natural apresentou uma tixotropia mais pronunciada do que a do amido 9460. De acordo com a
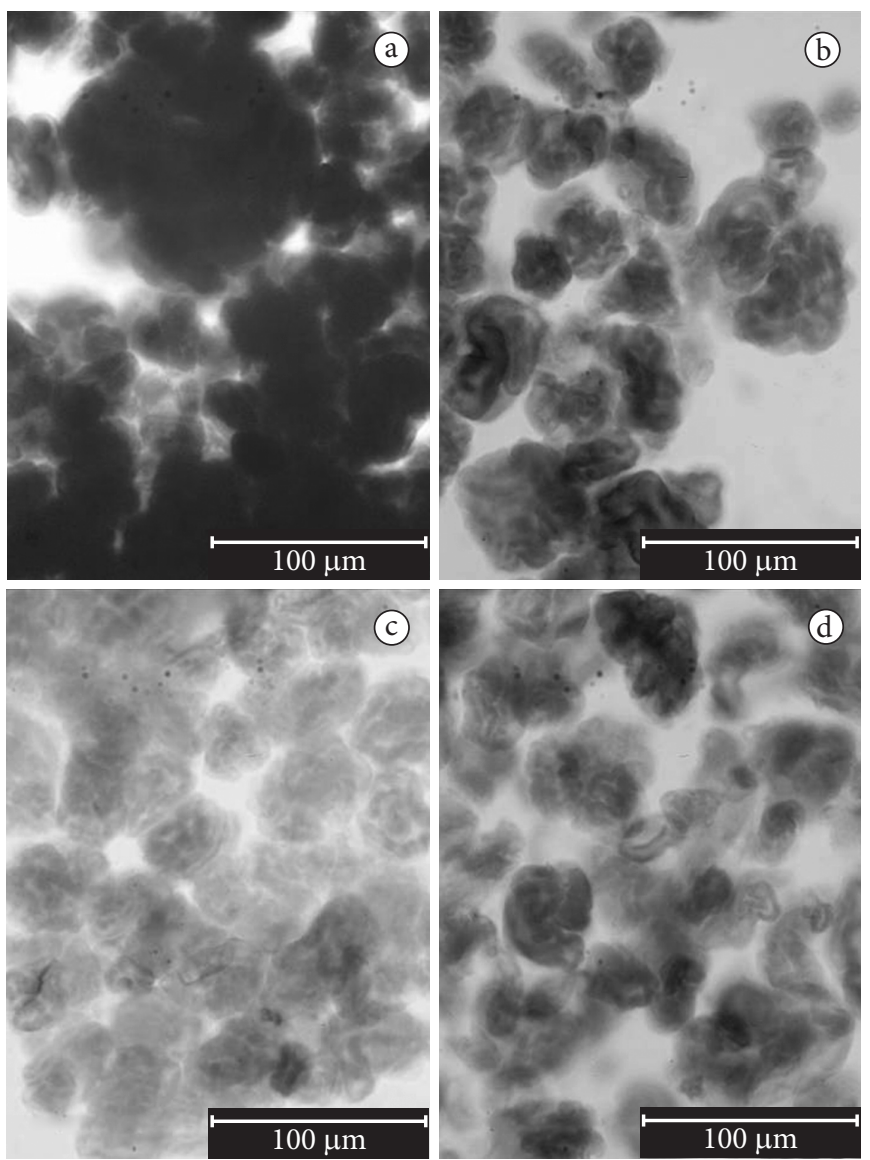

Figura 2. Grânulos dos amidos 9560 na temperatura de $55^{\circ} \mathrm{C}$ (a), aquecidos na temperatura de $95^{\circ} \mathrm{C}(\mathrm{B})$, na temperatura de $95^{\circ} \mathrm{C}$ em $\mathrm{pH}$ ácido (C) e depois de autoclavado (D). microscopia (Figuras 1 e 2), não há uma diferença significativa do tamanho dos grânulos dos amidos. Provavelmente, existem diferenças entre o arranjo dos grânulos na suspensão e na rigidez. Para o amido 9460, a acidez e o tratamento térmico aumentaram a tixotropia e, para o amido 9560, a acidez aumentou e o tratamento térmico diminuiu a tixotropia.

Tárrega, Véllez-Ruiz, Costell (2005) relataram que os géis dos amidos modificados de milho e mandioca, numa concentração de $4 \%$, apresentaram comportamento tixotrópico, com exceção do gel de amido de milho a $6 \%$, que apresentou reopexia. Outros autores reportaram a dependência do tempo dos amidos. Nguyen, Jensen, Kristensen (1998) encontraram que as dispersões de amido de milho normal ou ceroso apresentaram tixotropia e que o aumento da concentração não mostra um efeito significativo na característica tixotrópica da pasta, enquanto que Tecante et al. (1999), Nayouf, Loisel, Doublier (2003) observaram um comportamento antitixotrópico nas curvas de escoamento de pastas de amido de milho ceroso reticuladas. Os últimos

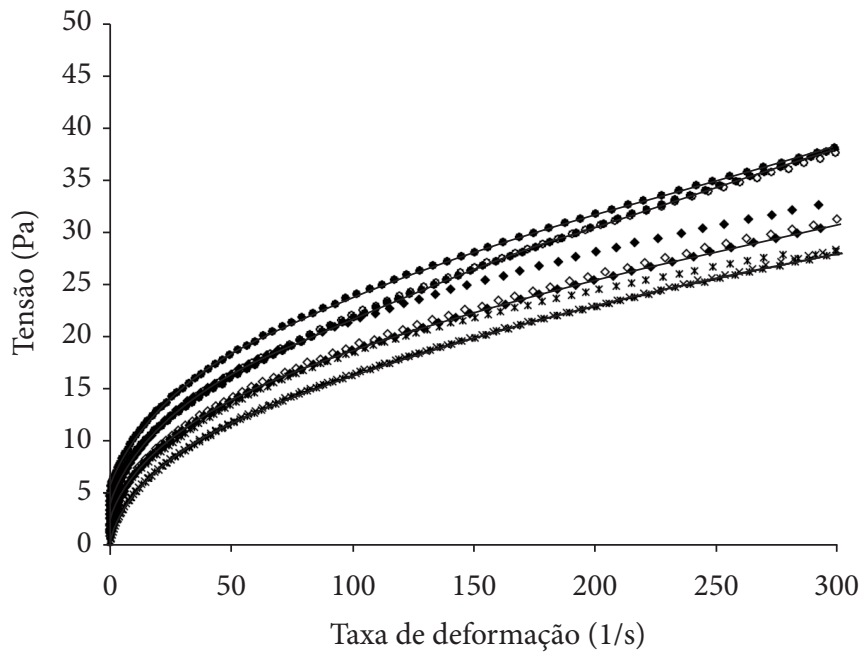

$\begin{array}{lll}\bullet \text { ac s1 } & \diamond \text { ac d } & - \text { ac s2 } \\ * \text { nat s1 } & \times \text { nat d } & * \text { nat s2 } \\ - \text { nat aut s1 } & \diamond \text { nat aut d } & \rightarrow \text { nat aut s2 }\end{array}$

Figura 3. Curva de escoamento dos géis dos amidos comerciais 9460 ácidos, naturais e autoclavados a $30^{\circ} \mathrm{C} . \mathrm{s} 1=$ subida $1 ; \mathrm{d}=$ descida; $\mathrm{s} 2=$ subida $2 ;$ ac $=$ ácido, nat $=$ natural, aut $=$ natural autoclavado.

Tabela 2. Média e desvio padrão dos parâmetros estimados das equações de Herschel-Bulkley dos géis dos amidos comerciais.

\begin{tabular}{|c|c|c|c|c|c|c|}
\hline Tratamento & $\mathrm{n}$ & $\mathrm{k}\left(\mathrm{Pa} \cdot \mathrm{s}^{\mathrm{n}}\right)$ & $\sigma_{\mathrm{o}}(\mathrm{Pa})$ & $\mathrm{R}^{2}$ & $\chi^{2}$ & Tixotropia $(\mathrm{Pa} / \mathrm{s})$ \\
\hline \multicolumn{7}{|c|}{9460} \\
\hline natural & $0,468^{\mathrm{a}} \pm 0,00$ & $2,001^{a} \pm 0,09$ & - & 0,999 & 0,84 & 150,44 \\
\hline ácido & $0,479^{\mathrm{b}} \pm 0,00$ & $1,973^{\mathrm{b}} \pm 0,01$ & $0,651^{a} \pm 0,11$ & 0,999 & 1,89 & 1828,86 \\
\hline natural autoclavado & $0,565^{c} \pm 0,00$ & $1,389^{c} \pm 0,12$ & $3,183^{b} \pm 0,46$ & 0,999 & 10,52 & 451,40 \\
\hline \multicolumn{7}{|c|}{9560} \\
\hline natural & $0,475^{\mathrm{d}} \pm 0,00$ & $2,016^{\mathrm{d}} \pm 0,14$ & - & 0,999 & 0,86 & 802,04 \\
\hline ácido & $0,455^{\mathrm{e}} \pm 0,00$ & $2,364^{\mathrm{e}} \pm 0,08$ & - & 0,999 & 1,89 & 1075,30 \\
\hline natural autoclavado & $0,548^{\mathrm{f}} \pm 0,00$ & $2,130^{\mathrm{f}} \pm 0,31$ & $4,13^{\mathrm{c}} \pm 0,82$ & 0,999 & 6,64 & 233,34 \\
\hline
\end{tabular}

$\mathrm{a}, \mathrm{b}, \mathrm{c}, \mathrm{d}, \mathrm{e}, \mathrm{f}-$ letras diferentes numa mesma coluna são estatisticamente diferentes para $\mathrm{p}<0,05$ 
autores interpretaram que o comportamento antitixotrópico poderia ser explicado por um empacotamento mais denso dos grânulos de amidos provocado pelo cisalhamento. Assim em suspensões concentradas de amidos cerosos com baixo conteúdo de amilose, as características reológicas estariam determinadas pela fração volumétrica do amido e sua deformabilidade. Tattiyakul, Rao (2000) observaram que uma dispersão a 5\% de amido reticulado exibiu um comportamento combinado das histereses. O comportamento antitixotrópico apareceu em baixas taxas de deformação e o tixotrópico em altas taxas. Entretanto vários autores determinaram comportamento tixotrópico para amido de milho ceroso reticulado (RAMASWANY et al., 1995) numa concentração de 3 e 4\% e para suspensões de suspensões de amido de milho normal (7\%) (HAN et al. 2002). Entretanto, Rao et al. (1997) relataram que dispersões de amidos de milho ceroso modificados, a $2,6 \%$ e temperatura de $20^{\circ} \mathrm{C}$, apresentaram comportamento reopético, ou seja, antitixotrópico.

\section{Curva de escoamento}

Nas Figuras 3 e 4, observa-se que a rampa descendente coincide com os dados obtidos na segunda rampa ascendente, assim, nestes dados foi eliminado o efeito da tixotropia. Nas Figuras 5a e $6 a$ são apresentados estes dados para os diversos tratamentos. Todos os amidos estudados apresentaram comportamento pseudoplástico. Este comportamento pode ser verificado nas Figuras $5 \mathrm{~b}$ e $6 \mathrm{~b}$, nas quais se apresenta a viscosidade aparente em função da taxa de deformação.

O gel do amido natural 9460 (Figura 5) apresentou valores mais baixos de tensão se comparados aos valores do gel ácido e aos do gel autoclavado. Assim, tanto o tratamento térmico como o ácido provocaram uma maior viscosidade do amido quando se observa a mesma taxa de deformação. O mesmo pode ser

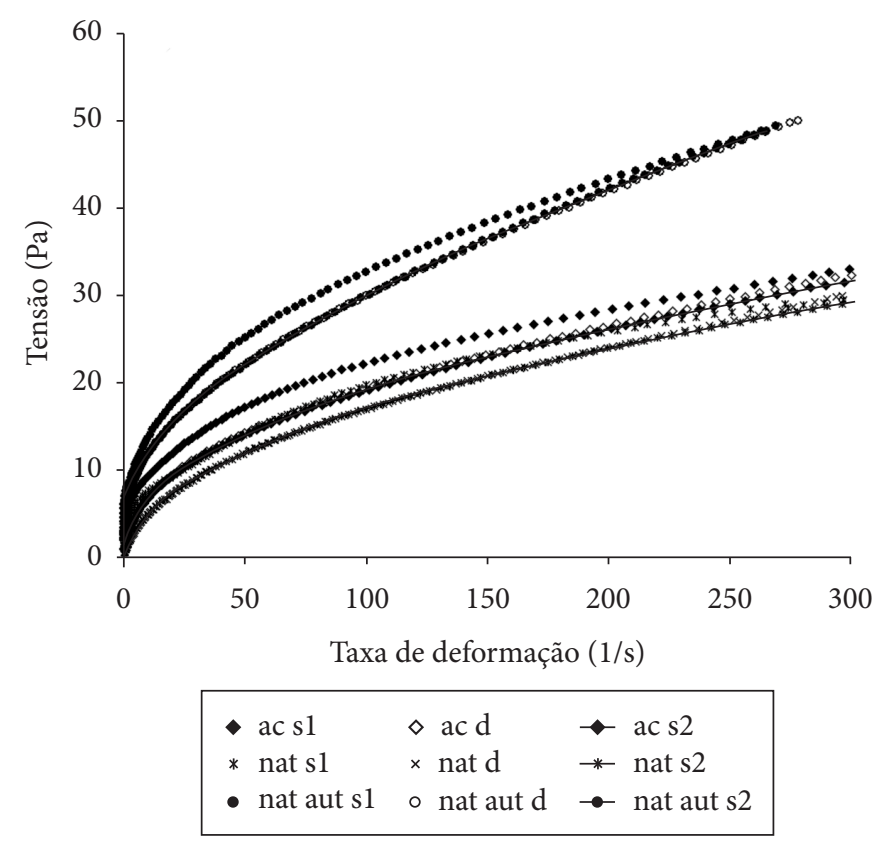

Figura 4. Curva de escoamento dos géis dos amidos comerciais 9560 ácidos, naturais e autoclavados a $30^{\circ} \mathrm{C} . \mathrm{s} 1=$ subida $1 ; \mathrm{d}=$ descida; $\mathrm{s} 2=$ subida $2 ; \mathrm{ac}=$ ácido, nat $=$ natural, aut $=$ natural autoclavado. observado na suspensão do amido 9560 (Figura 6). No entanto, o gel tratado termicamente apresentou valores mais elevados de tensão se comparado às suspensões ácidas.

Assim a adição do ácido cítrico e o tratamento térmico influenciaram o comportamento dos géis dos amidos 9460 e 9560 $(\mathrm{p}<0,05$,$) aumentando os valores de tensão medidos à taxa$ de deformação constante, se comparados aos dos géis naturais. Nas fotos da microscopia (Figuras 1 e 2) pode-se observar que o tratamento térmico provocou uma leve expansão dos grânulos e desagrupou os grânulos dos amidos, o que poderia explicar estas tendências.

Nguyen, Jensen e Kristensen (1998) estudaram o comportamento reológico de amido de milho normal e ceroso. O comportamento do gel observado nos amidos cerosos pode ser causado pela habilidade de expansão. Os grânulos de amidos inchados e gelatinizados podem estar tão próximos que a formação da estrutura de rede
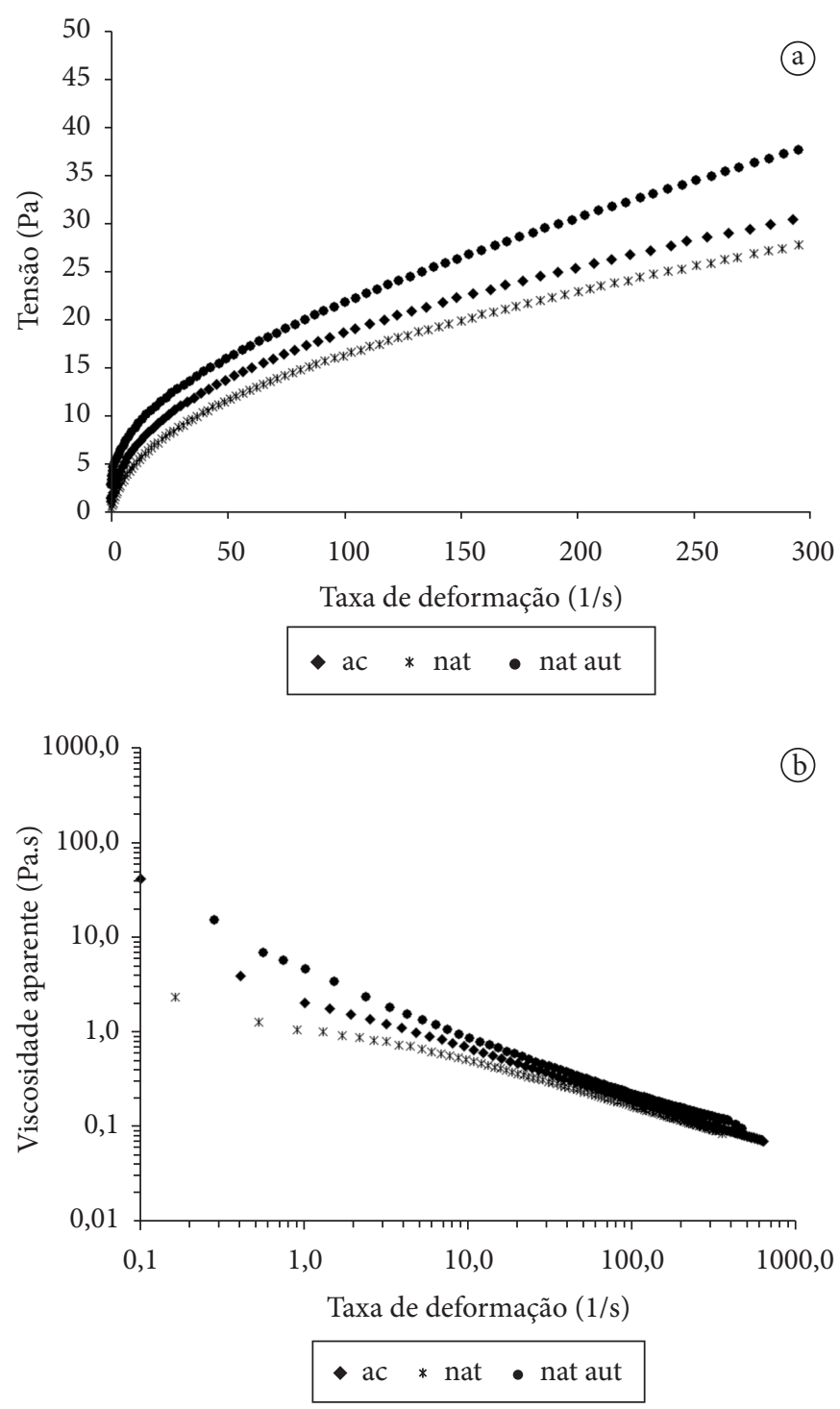

Figura 5. Curva de escoamento (a) e viscosidade aparente (b) dos géis dos amidos 9460 , na temperatura de $30^{\circ} \mathrm{C} . \mathrm{ac}=$ ácido, nat = natural, aut $=$ natural autoclavado. 
é facilitada pela associação de curtas ramificações externas das moléculas da amilopectina, enquanto o mecanismo de formação do gel de amidos regulares é diferente do gel de amido ceroso. Os autores indicaram que ambos os géis quebram sob cisalhamento, seguindo uma cinética estrutural de terceira ordem. Isto sugere que os tipos de estrutura dos amidos de milho normal e ceroso quebram por uma ruptura de ponte da rede do mesmo tipo, que podem ser, simplesmente, as pontes de hidrogênio dos grupos hidroxilas nas cadeias de amilose adjacentes no amido de milho normal. A taxa de quebra da estrutura é mais rápida no amido normal do que no amido ceroso, como pode ser visto nos dados de viscosidade e tixotropia, em que a viscosidade do amido normal apresentou-se mais alta no início e depois ficou menor se comparada ao amido ceroso (NGUYEN JENSEN; KRISTENSEN, 1998). Isto pode indicar que as ligações da rede encontradas nas cadeias externas de amilopectina no amido de
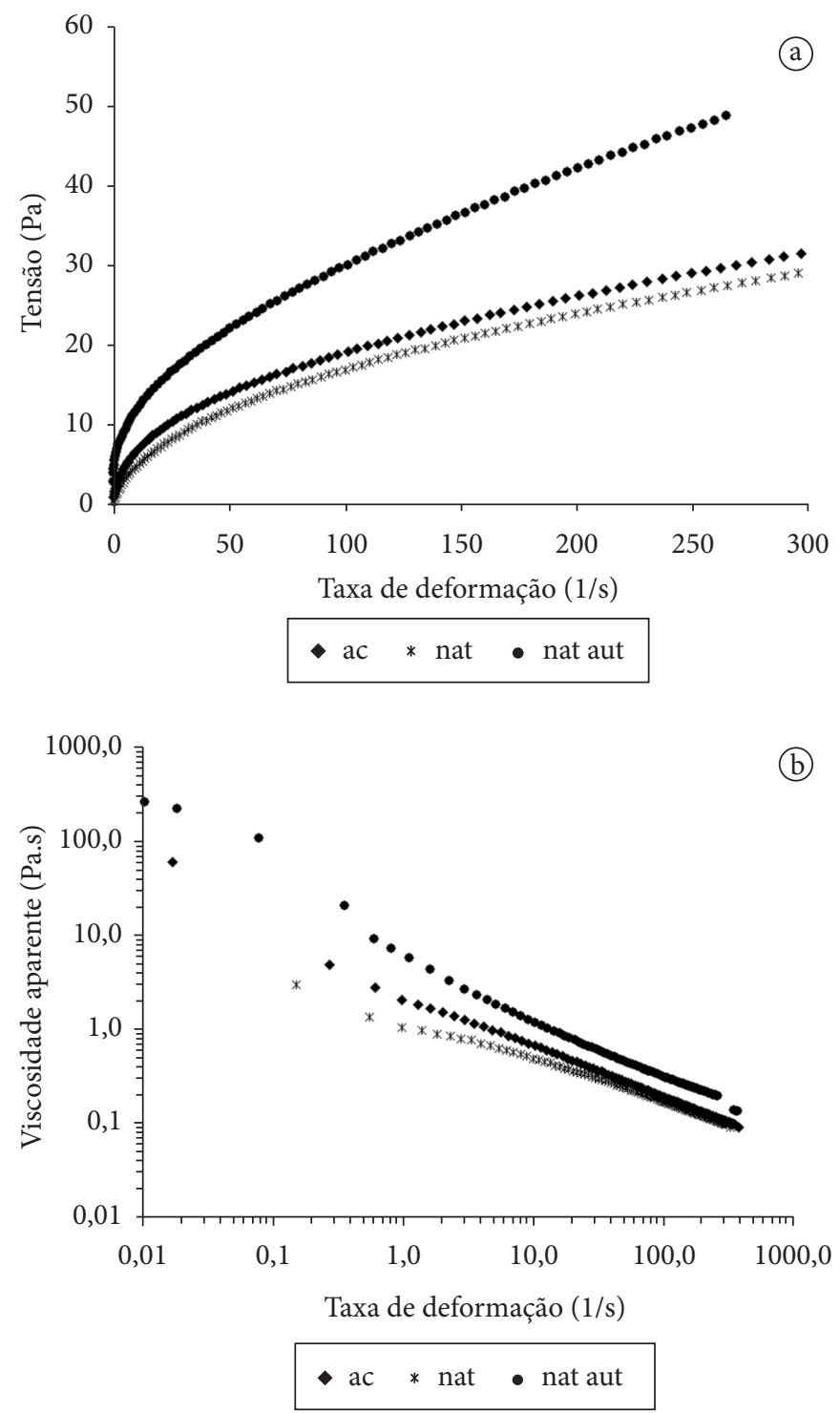

Figura 6. Curva de escoamento (a) e viscosidade aparente (b) dos géis dos amidos 9560 , na temperatura de $30^{\circ} \mathrm{C}$. ac = ácido, nat = natural, aut $=$ natural autoclavado. milho ceroso são poucas e mais rígidas do que aquelas existentes nas cadeias de amilose linear no amido de milho normal.

\section{Modelagem matemática das curvas de escoamento}

A modelagem matemática das curvas de escoamento foi realizada utilizando-se o modelo de Herschel-Bulkley. Os valores dos parâmetros estimados para as suspensões dos amidos e para os diversos tratamentos são apresentados na Tabela 2, na qual os valores dos parâmetros apresentados resultaram do melhor ajuste obtido. Pode-se observar que, para alguns tratamentos, o comportamento dos géis foi mais bem descrito pela Lei da Potência. Bhandari, Singhal, Kale (2002) relataram que os géis dos amidos de milho, nas concentrações de 3 e 5\%, apresentaram um comportamento mais bem descrito pela equação Lei da Potência.

Os valores de $(n)$ foram maiores para os géis naturais autoclavados, independente do amido. Para o amido 9560, o gel ácido apresentou menor valor de (n), se comparado ao gel natural. Os valores de $(\mathrm{k})$ foram altos para todos os géis, no entanto, os valores foram diminuindo para o gel ácido e o gel autoclavado, para o gel 9460. Para o gel 9560, o gel ácido apresentou o maior valor de (k). Houve influência da acidez e do tratamento térmico $(\mathrm{p}<0,05)$ para os valores de $(\mathrm{n}),(\mathrm{k})$ e $\left(\sigma_{\mathrm{o}}\right)$.

\section{Ensaios oscilatórios (espectro mecânico)}

Os espectros mecânicos obtidos por reologia dinâmica são uma das medidas mais comuns para o estudo do comportamento visco-elástico de alimentos porque os resultados obtidos são muitos sensíveis à composição química e à estrutura física (STEFFE, 1996). O gel de amido pode ser considerado como um sistema constituído de grânulos inchados e embebidos numa rede tridimensional de cadeias de amilose agregadas (AHMAD; WILLIANS, 2001). As propriedades reológicas do sistema são determinadas por uma fração do volume da fase dispersa (grânulos remanescentes de amilopectina), pela reologia da fase contínua (amilose lixiviada) e pela interação entre essas fases.

Nas Figuras 7A e 8A pode-se observar que as suspensões dos amidos comerciais apresentaram a curva de $\left(G^{\prime}\right)$ superior a curva de (G”), mostrando um comportamento de gel elástico. As Figuras 7B e 8 B apresentam o comportamento de Tan $\delta\left(G^{\prime \prime} / G^{\prime}\right)$, que aumentou com a frequência.

As curvas de (G') e (G”), das Figuras 7A e 8A, apresentaram uma pequena dependência com a frequência. Segundo Steffe (1996), Chamberlain, Rao (2000), géis fracos ou géis físicos são dependentes da frequência, apresentam curva de $\left(G^{\prime}\right)$ maior que (G”), mas não apresentam cruzamento de $\left(G^{\prime}\right)$ e (G”). Em géis elásticos ou verdadeiros, os módulos de armazenamento não variam com a frequência. A diferença de um gel verdadeiro, um gel fraco em altas deformações (ensaios estacionários), escoa, mas um gel verdadeiro sofre ruptura (IKEDA; NISHINARI, 2001).

Todos os géis, nas condições estudadas, apresentaramse como géis elásticos fracos e apresentaram influência nos tratamentos nos valores de $\left(G^{\prime}\right)$ e $\left(G^{\prime \prime}\right)$, para $p<0,05$. Os géis ácidos dos amidos 9460 apresentaram valores maiores de G', 
seguidos do gel natural autoclavado e do gel natural. Para os géis dos amidos 9560, também o gel ácido apresentou os maiores valores de G', seguido do gel natural e natural autoclavado. Observando-se os valores de Tan $\delta$ (Figuras $7 \mathrm{~b}$ e $8 \mathrm{~b}$ ) também pode verificar-se que o gel ácido é levemente mais estruturado e que o tratamento térmico provocou um ligeiro aumento dos valores de Tan $\delta$, ou seja, de perda do caráter visco-elástico. Hirashima, Takahashi e Nishinari (2005) verificaram a influência do pH na suspensão de amido de milho durante o aquecimento a $97^{\circ} \mathrm{C}$, com vários ácidos fracos (acético, ascórbico, cítrico, láctico, tartárico e málico). Suspensões de amido a $\mathrm{pH}$ próximos a 6 comportaram-se como suspensões emaranhadas, mas quando o pH de tratamento foi fixado na faixa de 4-5, as suspensões tiveram um comportamento de gel fraco, com um aumento considerável de G. Este comportamento coincidiu com uma

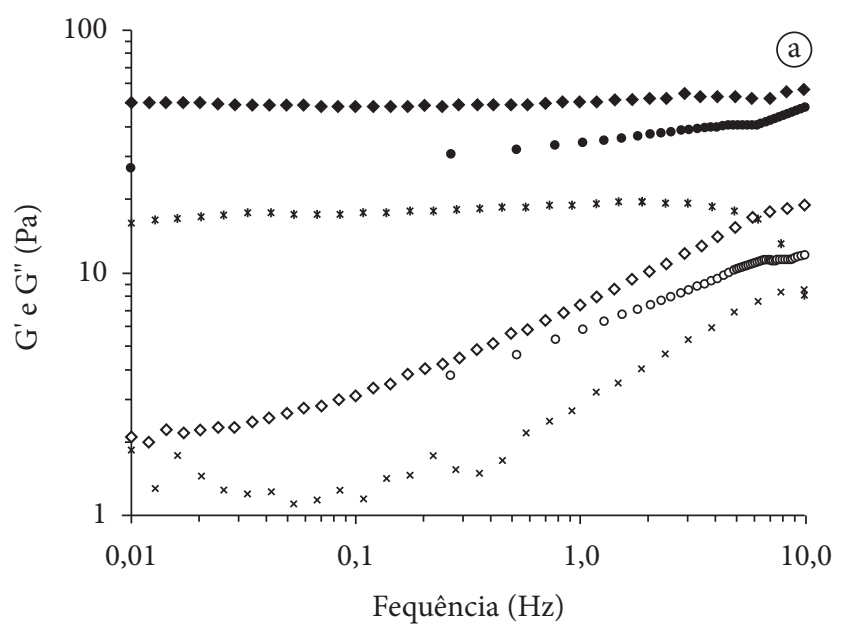

$\begin{array}{ll}\bullet \text { ac } G^{\prime} & \diamond \text { ac } G^{\prime \prime} \\ * \text { nat } G^{\prime} & \times \text { nat } G^{\prime \prime} \\ \bullet \text { nat aut } G^{\prime} & \diamond \text { nat aut } G^{\prime \prime}\end{array}$

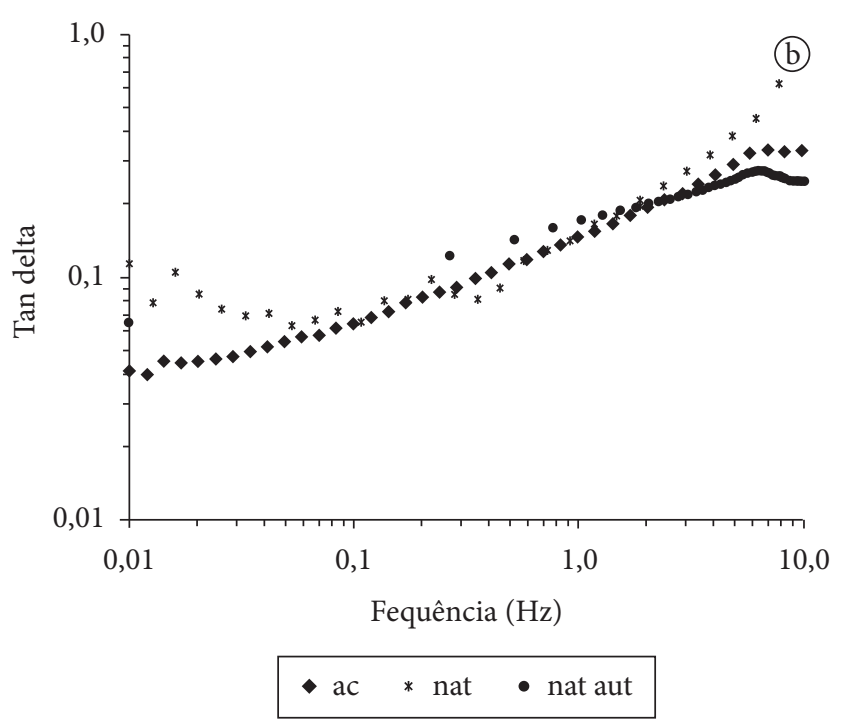

Figura 7. Espectro mecânico (a) e tan $\delta$ (b) dos géis do amido 9460 na temperatura de $30^{\circ} \mathrm{C}$. ac = ácido, nat $=$ natural, nat aut = natural autoclavado. leve queda da viscosidade intrínseca. Para $\mathrm{pH}$ de 3,5, a queda de viscosidade intrínseca foi muito abrupta e a suspensão mostrou novamente características mais viscosas que elásticas $(G$ ” < G'). Assim, pôde-se concluir que o tratamento em meio ácido débil provocou a hidrólise do amido, mas, para certas faixas de $\mathrm{pH}$, o aumento do número de cadeias lixiviadas, mantendo o diâmetro dos grânulos sem grandes mudanças, provocou um aumento de viscosidade e do caráter visco-elástico da suspensão. Entretanto quando a hidrólise resultou num decréscimo expressivo da massa molecular das cadeias de amilose e amilopectina, a viscosidade caiu e a suspensão assumiu um comportamento viscoso. Consequentemente, em condições não muito rigorosas de tratamento ácido, pode constatar-se um estruturação da suspensão de amido.

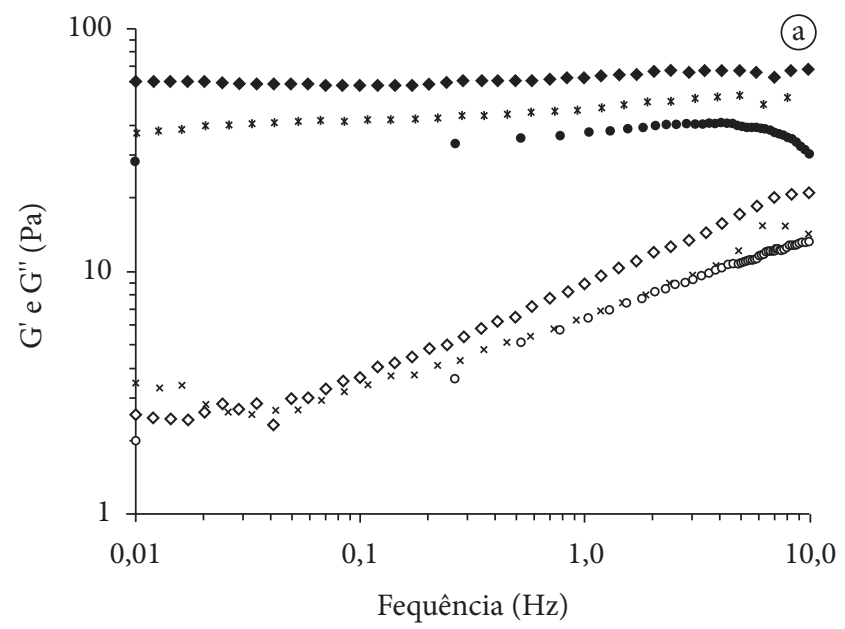

\begin{tabular}{ll|}
\hline ac $\mathrm{G}^{\prime}$ & $\diamond$ ac $\mathrm{G}^{\prime \prime}$ \\
$*$ nat $\mathrm{G}^{\prime}$ & $\times$ nat $\mathrm{G}^{\prime \prime}$ \\
$\bullet$ nat aut $\mathrm{G}^{\prime}$ & $\diamond$ nat aut $\mathrm{G}^{\prime \prime}$ \\
\hline
\end{tabular}

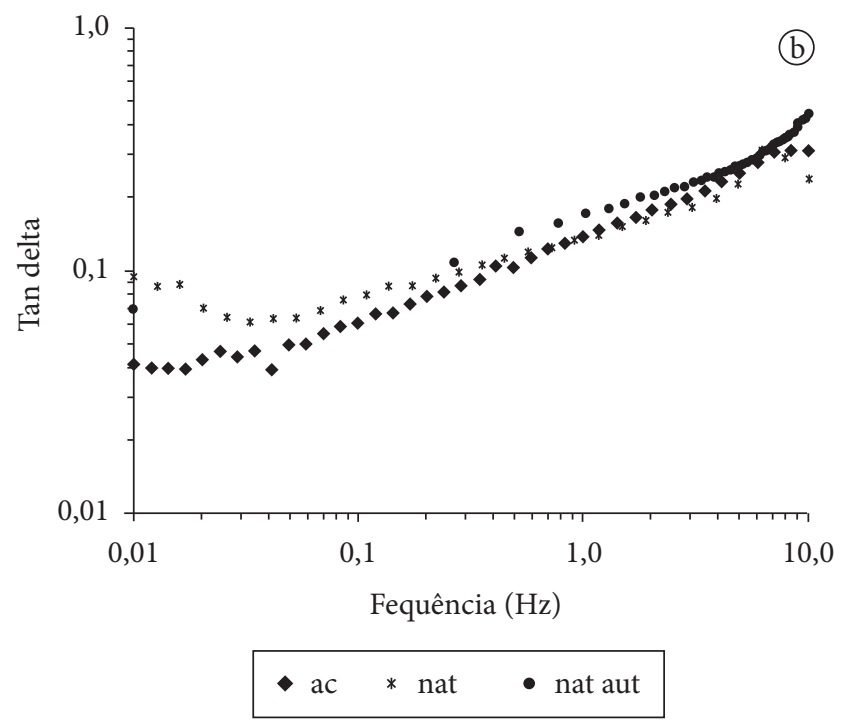

Figura 8. Espectro mecânico (a) e tan $\delta$ (b) dos géis do amido 9560 na temperatura de $30^{\circ} \mathrm{C}$. ac $=$ ácido, nat $=$ natural, nat aut $=$ natural autoclavado. 


\section{Conclusões}

Neste trabalho foi estudada a influência do tratamento térmico e da acidez no comportamento reológico dos amidos de milho ceroso nativos funcionais orgânicos comerciais pré-gelatinizados. A microscopia revelou que a acidez e o tratamento térmico não modificaram a estrutura dos grânulos dos amidos, mas podem ter induzido a uma leve expansão dos grânulos, refletida no comportamento reológico dos amidos, que apresentaram resistência ao tratamento térmico e à acidez, estruturando ainda mais os géis.

Os géis apresentaram comportamento não newtoniano, pseudoplástico, modelado pela equação Lei da Potência para o gel natural do amido 9460 e para o gel natural e ácido do amido 9560. Os géis ácido e natural autoclavado do amido 9460 e o natural autoclavado do amido 9560 foram modelados pela equação Herschel-Bukley. Todos os géis apresentaram comportamento de gel elástico fraco.

\section{Agradecimentos}

Ao CNPq pelo auxílio através da bolsa de doutorado.

À National Starch and Chemical Industrial, através de Andréa, pela doação dos amidos.

\section{Referências bibliográficas}

AHMAD, F. B.; WILLIANS, P. A. Effects of galactomannans on the thermal e rheological properties of sago starch. Journal of Agricultural and Food Chemistry, v. 49, n. 3, p. 1578-1586, 2001.

ASSOCIATION OF OFFICIAL ANALYTICAL CHEMISTS - AOAC. Official Methods of Analysis. 16 ed. Washington, 1997.

ASSOCIATION OF OFFICIAL ANALYTICAL CHEMISTS - AOAC. Official Methods of Analysis. Washington, 1975.

BHANDARI, P. N.; SINGHAL, R. S.; KALE, D. D. Effect of succinylation on the rheological profile of starch pastes. Carbohydrate Polymers, v. 47, n. 3, p. 365-371, 2002.

BRETON-DOLLET, V. Influence du couple temperature/cisaillemet sur les propriétés rhéologiques de preparations à base d'almidon. Villeneuve d'Ascq, France, 1996. These (Doctorat) - INRA.

ELLIS, R. P. et al. Starch production and industrial use. Journal of Science Food Agriculture, v. 77, p. 289-311, 1998.

$\mathrm{HAN}, \mathrm{X}$. et al. Influence of maize starch granule-associated protein on the rheological properties of starch pastes. Part I. Large deformation measurements of paste properties. Carbohydrate Polymers, v. 49, n. 3, p. 315-321, 2002.

HIRASHIMA, M.; TAKAHASHI, R.; NISHINARI, K. Effects of adding acids before and after gelatinization on the viscoelasticity of cornstarch pastes. Food Hydrocolloids, v. 19, n.5, p. 909-914, 2005.

IKEDA, S.; NISHINARI, K. "Weak-gel" type rheological properties of aqueus dispersions of nonaggregated $r$-carrageenan helices. Journal of Agricultural and Food Chemistry, v. 49, n.9, p. 4436-4441, 2001.

JULIANO, B. O. A simplified assay for milled-rice amylose. Cereal Science Today, v. 16, n. 10, p. 334-340, 1971.

KUHN, K.; SCHLAUCH, S. Comparative study about commercially available starches for high shear and high temperature applications in food. Starch/Stärke, v. 46, n. 6, p. 208-218, 1994.

LAGARRIGUE, S.; ALVAREZ, G. The rheology of starch dispersions at high temperatures and high rates: a review. Journal of Food Engineering, v. 50, n. 4, p. 189-202, 2001.
MARQUES, P. T. et al. Study of gelatinization process and viscoelastic properties of cassava starch: effect of sodium hydroxide and ethylene glycol diacrylate as cross-linking agent. Carbohydrate Polymers, v. 66, n. 3, p. 396-407, 2006.

MARTINEZ, C.; CUEVAS, F. Evalución de la calidad culinaria y molinera del arroz. Guia de estudo do Centro Internacional de Agricultura Tropical - CIAT, 1989.

NAYOUF, M.; LOISEL, C.; DOUBLIER, J. L. Effect of thermomechanical treatment on the rheological properties of crosslinked waxy corn starch. Journal of Food Engineering, v. 59, n. 2-3, p. 209-219, 2003.

NURUL, I. M.; AZEMI, B. M. N. M.; MANAN, D. M. A. Rheological behavior of sago (Metroxylon sagu) starch paste. Food Chemistry, v. 64, n. 4, p. 501-505, 1999.

NGUYEN, Q. D.; JENSENAND, C. T. B.; KRISTENSEN, P. G. Experimental and modelling studies of the flow properties of maize and waxy maize starch pastes. Chemical Engineering Journal, v. 70, n. 2, p. 165-171, 1998.

RAMASWANY, H. S. et al. Rheological properties of gelatinized starch solutions as influenced by thermal processing in an agitating retort. Journal of Food Engineering, v. 25, n. 3, p. 441-454, 1995.

RAO, M. A. et al. Rheological behaviour of heated starch dispersions in excess water:role of starch granule. Carbohydrate Polymers, v. 33, n. 4, p. 273-283, 1997.

SANDERSON, G. P. Polysaccharides in Foods. Food Technology, v. 7 , n. 83, p. $50-57,1981$.

SINGH, N. et al. Morphological, thermal and rheological properties of starches from different botanical sources. Food Chemistry, v. 81, n. 2, p. 219-231, 2003.

STEFFE, J. F. Rheological Methods in Food Process Engineering. 2 ed. Michigan: Freeman Press, 1996. 418 p.

SRIBURI, P.; HILL, S. E. Extrusion of cassava starch with either variations in ascorbic acid concentration or $\mathrm{pH}$. International Journal of Food Science and Technology, v. 35, n. 2, p. 141-154, 2000.

SRIBURI, P.; HILL, S. E.; MITCHELL, J. R. Effects of ascorbic acid on the conversion of cassava starch. Food Hydrocolloids, v. 13, n. 2, p. 177-183, 1999.

TAKEDA, Y. et al. Structures of branched molecules of amyloses of various origins and molar fractions of branched and unbranched molecules. Carbohydrate Research, v. 165, n. 1, p. 139-145, 1987.

TARREGA, A.; VÉLEZ-RUIZ, J. F.; COSTELL, E. Influence of milk on the behaviour of cross-linked waxy maize and tapioca starch dispersion. Food Research International, v. 38, n.7, p. 759-768, 2005.

TATTIYAKUL, J.; RAO, M. A. Rheological bahavior of cross-linked waxy maize starch dispersion during and after heating. Carbohydrate Polymers, v. 43, n. 3, p. 215-222, 2000.

TECANTE, A.; DOUBLIER, J. L. Steady flow and viscoelasti behaviour of crosslinked waxy corn starch $\mathrm{k}$-carrageenan pastes and gels. Carbohydrate Polymers, v. 40, n. 3, p. 221-231, 1999.

VALLÉS-PÀMIES, B. et al. The effects of low molecular weight additives on the viscosities of cassava starch. Carbohydrate Polymers, v. 34, n.1-2, p. 31-38, 1997.

WANG, L.; WANG, Y. Structure and physicochemical properties of acid-thinned corn, potato and rice starches. Starch/Stärke, v. 53, n. 11, p. 570-576, 2001.

YAMADA, T.; MORIMOTO, Y.; HISAMATSU, M. Effects of citric acid on potato starch gelatinization. Starch/Stärke, v. 38 , n. 8 , p. 264-268, 1986.

ZOBEL, H. F. Molecules to granules: a comprehensive starch review. Starch/Stärke, v. 40, n. 2, p. 44-50, 1988. 